\section{Lipid Abnormalities Induced by Novel Antipsychotic Drugs}

The authors reply:

We would like to thank $\mathrm{Dr} \mathrm{Su}$ et al. for their valuable comments. We share their opinion that more attention should be paid to the possible effects of new antipsychotic drugs on serum lipid and especially triglyceride levels. However, we feel that routine measurement of the lipid profile during treatment with antipsychotic drugs should be further specified. Barth et al. ${ }^{[1]}$ assessed the need for routine measurements of plasma lipids in patients treated with isotretinoin, a drug which is also known to increase triglyceride levels. They found that determination of the lipid profile prior to onset of therapy and on one occasion after 4 weeks of therapy was sufficient. More frequent determinations should be saved for patients predisposed to hypertriglyceridaemia and patients with diabetes mellitus. We propose the same recommendations for the measurement of serum lipid levels in patients starting with atypical antipsychotic drugs.

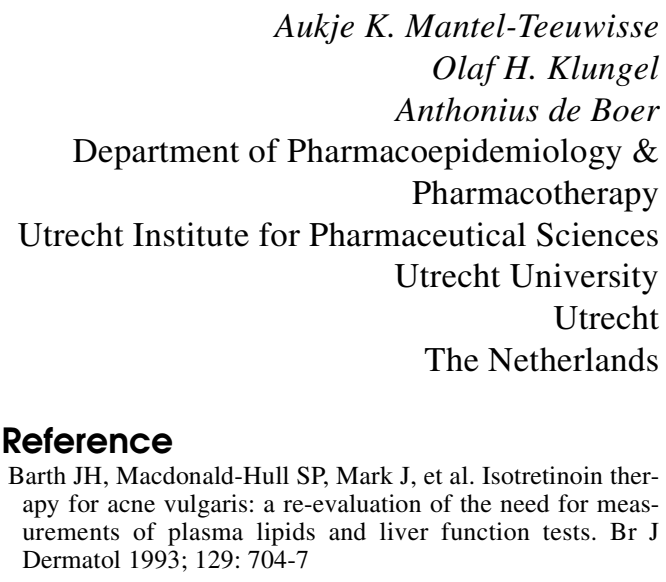
Olaf H. Klungel coepidemiology \& Sciences Utrecht Dermatol 1993; 129: 704-7 arth JH, Macdonald-Hull SP, Mark J, et al Isotretinoin ther- 